\title{
Contribuição do guaraná em pó (Paullinia cupana) como fonte de cafeína na dieta
}

\section{Contribution of guaraná powder (Paullinia cupana) as a source of caffeine in the diet}

\author{
Sílvia Amélia Verdiani TFOUNI ${ }^{1}$ \\ Mônica Cristiane Rojo CAMARGO ${ }^{1}$ \\ Sílvia Helena Pereira VITORINO ${ }^{2}$ \\ Thaís Feres MENEGÁRIO³ \\ Maria Cecília de Figueiredo TOLEDO²
}

\section{RE S U M O}

\section{Objetivo}

Este estudo teve como objetivo determinar os teores de cafeína em diferentes marcas de guaraná em pó disponíveis comercialmente.

\section{Métodos}

A metodologia analítica utilizada envolveu as etapas de extração com água, limpeza da amostra com acetato de chumbo e determinação por cromatografia líquida de alta eficiência com detector de arranjo de diodos (200-400nm). Foram analisadas 39 amostras adquiridas nas cidades de Campinas e Ribeirão Preto, SP, entre dezembro de 2003 e janeiro de 2004.

\section{Resultados}

Os teores de cafeína nas amostras apresentaram grande variabilidade, situando-se na faixa de 9,52 a 36,71mg/g de pó, isso se deve, possivelmente, às diferenças de procedência e processo a que a matéria-prima foi submetida. Comparando-se com o pó de café, fonte tradicional de cafeína na dieta, verifica-se que o teor médio de cafeína encontrado no guaraná em pó, dependendo da marca considerada, pode ser até quatro vezes maior.

\section{Conclusão}

Entre os consumidores de guaraná em pó esse produto pode ser considerado uma importante fonte de cafeína na dieta, e sua associação com demais produtos que contêm cafeína sugere que seu consumo deve ser controlado, uma vez que controvérsias persistem quanto à dose segura de ingestão da cafeína.

Termos de indexação: cafeína; cromatografia líquida de alta eficiência; guaraná.

\footnotetext{
1 Instituto de Tecnologia de Alimentos, Centro de Pesquisa e Desenvolvimento de Química de Alimentos e N utrição Aplicada. Av. Brasil, n. 2880, 13070-178, Campinas, SP, Brasil. Correspondência para/Correspondence to: S.A.V. TFOUNI. E-mail: <founi@ital.sp.gov.br>.

2 Universidade Estadual de Campinas, Faculdade de Engenharia de Alimentos, Departamento de Ciência de Alimentos. Campinas, SP, Brasil.

${ }^{3}$ Pontifícia Universidade Católica de Campinas. Centro de Ciências da Vida, Faculdade de Ciências Farmacêuticas. Campinas, SP, Brasil
} 


\section{A B S T R A C T}

\section{Objective}

The present study was conducted in order to determine the caffeine levels in different brands of commercially available guaraná powder.

\section{Methods}

The analytical methodology involved extraction with water, clean-up with saturated basic lead acetate solution and determination by high performance liquid chromatography with diode array detector (200-400nm). 39 samples were purchased in the cities of Campinas and Ribeirão Preto, SP, between December 2003 and January 2004 and analyzed.

\section{Results}

The caffeine levels in the samples varied widely ranging from 9.52 to $36.71 \mathrm{mg} / \mathrm{g}$, probably due to differences in the origin of the raw material and to the types of processing that the guaraná seeds are submitted to. In comparison to coffee, traditional source of caffeine in the diet, it is possible to verify that the average caffeine content in guaraná powder is about four times higher than the quantities present in coffee.

\section{Conclusion}

Among guaraná powder consumers, this product may be considered as an important source of caffeine in the diet. The association with other products containing caffeine suggests that the guaraná powder consumption should be controlled as the debate regarding caffeine's safe dose of intake remains.

Indexing terms: caffeine; high performance liquid chromatography; guaraná.

\section{N T R O D U ÇÃ O}

A cafeína, um derivado metilado de bases purínicas estruturalmente identificada como 1,3,7-trimetilxantina, é considerada como a substância psicoativa mais consumida em todo 0 mundo, por pessoas de todas as idades, independentemente do sexo e da localização geográfical-4. Esse alcalóide está presente na natureza em mais de 63 espécies de plantas, entre elas, o guaranazeiro, que apresenta os maiores teores de cafeína, principalmente nas sementes.

A relação entre o consumo de cafeína e o possível desenvolvimento de algumas doenças tem despertado, há muito tempo, o interesse de pesquisadores. Embora muitas pesquisas tenham sido feitas, ainda não existem evidências de que quantidades moderadas de cafeína (aproximadamente $300 \mathrm{mg} / \mathrm{dia}$ ) sejam prejudiciais à saúde de um indivíduo normal5,6. No entanto, um consumo superior a $400 \mathrm{mg}$ por dia pode levar ao chamado "cafeinismo", cujos sintomas mais comuns são ansiedade, inquietação, irritabilidade, tremores, perda de apetite, tensão muscular e palpitações no coração ${ }^{1,2,4-11}$.
Café, chá, produtos de chocolate e alguns refrigerantes são considerados mundialmente como as principais fontes de cafeína na dieta2,3,5,12,13. Secundariamente, o mate, o guaraná e vários medicamentos, como emagrecedores, diuréticos, estimulantes e analgésicos, também contribuem para a sua ingestão $0^{6,12}$.

Entende-se por guaraná os frutos extraídos de Paullinia cupana H.B.K. Typica e Paullinia cupana variedade sorbilis (M art.) Ducke, ambas pertencentes à família das Sapindáceas e nativas da Amazônia. 0 guaraná em pó, forma como o produto normalmente é comercializado, é resultante da semente finamente triturada, moída ou pilada após secagem. 0 Brasil é praticamente 0 único país a produzir guaraná em escala comercial em cultivos racionais e sistemáticos. Os principais estados produtores são Bahia, Amazonas, Mato Grosso, Acre e Pará14. A quantidade de cafeína no guaraná em pó pode variar de acordo com a procedência da matéria-prima (região de plantio), o método de cultivo, presença de contaminantes químicos e métodos de secagem ${ }^{15}$.

Entre as várias técnicas disponíveis para a análise de cafeína em alimentos, a cromatografia 
líquida de alta eficiência (CLAE) tem sido amplamente utilizada, em diversas matrizes, devido à sua rapidez e eficiência ${ }^{16-26}$.

A pesar de o guaraná ser conhecido e utilizado pelos indígenas brasileiros há muito tempo, não existe na literatura dados disponíveis quanto aos níveis de cafeína em guaraná em pó. Dessa forma, o presente estudo teve como objetivo determinar os teores de cafeína em diferentes marcas de guaraná em pó disponíveis comercialmente.

\section{M ÉTO D O S}

Amostras de guaraná em pó (13 marcas disponíveis, 3 lotes de cada) foram adquiridas no comércio das cidades de Campinas e Ribeirão Preto, SP, entre dezembro de 2003 e janeiro de 2004. Todas as amostras, num total de 39 , foram analisadas em duplicata.

A metodologia analítica utilizada para a determinação de cafeína em guaraná em pó foi aquela proposta por Camargo \& Toledo ${ }^{20}$ para análise de cafeína em pó de café, como o descrito a seguir:

Pesaram-se $10 \mathrm{~g}$ de pó de guaraná num béquer de $250 \mathrm{~mL}$, que foram misturados a $200 \mathrm{~mL}$ de água destilada. A solução foi filtrada por gravidade em um funil de papel com papel de filtro. Em seguida, tomou-se uma alíquota de $20 \mathrm{~mL}$ do filtrado e procedeu-se à limpeza da amostra. Adicionaram-se $6 \mathrm{~mL}$ de uma solução saturada de acetato de chumbo básico, centrifugando-se as amostras a 1057,5 $\mathrm{xg}$ por minuto durante 5 minutos. Retirou-se 0 sobrenadante e nele adicionou-se bicarbonato de sódio $(0,1 \mathrm{~g} / 10 \mathrm{~mL}$ água). A solução resultante foi novamente centrifugada por 5 minutosa $1057,5 \times$ g por minuto. Ao sobrenadante foi adicionado $\mathrm{HCl}$ (ácido clorídrico) 1,0 M, de modo a obter um pH ácido (ao redor de 4). Essa solução foi então completada com água destilada para $100 \mathrm{~mL}$ num balão volumétrico e injetada no cromatógrafo.

As análises foram feitas por CLAE. Para tanto, foi utilizado um equipamento Waters constituído por uma bomba quaternária modelo 600 , injetor automático modelo 717 , acoplado a um detector de arranjo de diodos modelo 996 (deteç̧ão e quantificação da cafeína a 272nm). Para a separação da cafeína foi empregada uma coluna cromatográfica Novapack C18 (150 x $3,9 \mathrm{~mm}$ d.i., partículas de $4 \mu \mathrm{m}$ ). A fase móvel utilizada na separação foi composta de acetonitrila-água $(10: 90, v / v)$, a uma vazão constante de $1,0 \mathrm{~mL} / \mathrm{min}$. 0 volume de injeção foi de $20 \mu \mathrm{L}$.

0 método utilizado para a quantificação de cafeína nas amostras foi o da padronização externa. A partir de uma solução estoque contendo $1,0 \mathrm{mg} / \mathrm{mL}$ de cafeína, obteve-se por diluição com água a faixa de concentrações desejadas $(0,04$ a $0,5 \mathrm{mg} / \mathrm{mL}$ ). A curva de calibração traçada mostrou-se linear dentro das concentrações de trabalho. 0 limite de detecção (expresso como concentração) foi determinado conforme proposto por Miller \& Miller ${ }^{27}$.

A identificação da cafeína nas amostras foi efetuada por comparação dos tempos de retenção dos picos de interesse e de seus respectivos espectros de absorção (200-400nm), com aqueles obtidos para o padrão nas mesmas condições de análise.

Os testes de recuperação foram realizados adicionando-se soluções padrão de cafeína em uma das amostras de guaraná em pó. As fortificações, em três níveis de concentração $(10 \mathrm{mg} / 100 \mathrm{~mL}$, $15 \mathrm{mg} / 100 \mathrm{~mL}$ e $20 \mathrm{mg} / 100 \mathrm{~mL}$ ), foram feitas em duplicata. As recuperações foram calculadas pela diferença entre a concentração dos compostos nas amostras fortificadas e não-fortificadas. Os resultados analíticos reportados não foram corrigidos em função da porcentagem de recuperação.

\section{RES U LT A D O S}

A Tabela 1 apresenta os valores médios obtidos nos testes de recuperação para os três níveis de fortificação. Esses valores se situaram na faixa de $71,5 \%$ a $80,5 \%$. Como pode ser observado a partir desses resultados, não houve perdas significativas durante o procedimento de 
extração. 0 limite de detecção para a cafeína foi de $0,04 \mathrm{mg} / \mathrm{mL}$. Os cromatogramas obtidos para (a) padrão de cafeína e para (b) uma amostra de guaraná em pó, nas condições de análise, estão ilustrados na Figura 1. Pode-se observar no cromatograma da amostra a boa separação do pico da cafeína, sem compostos interferentes.

A Tabela 2 apresenta os teores médios de cafeína nas diversas marcas de guaraná em pó e a faixa de concentração relativa aos 3 lotes analisados. A concentração média de cafeína encontrada nas amostras situou-se na faixa de 14,18 a $28,79 \mathrm{mg} / \mathrm{g}$ de pó.

\section{I S C U S S Ã O}

Em função dos resultados obtidos para 0 limite de detecção, nos testes de recuperação, $e$ da boa resolução do pico de interesse, pode-se considerar que o procedimento de análise adotado ${ }^{20}$ mostrou-se adequado para a determinação de cafeína em guaraná em pó. A adição do acetato de chumbo básico durante a extração proporcionou a obtenção de uma solução totalmente
Tabela 1. Valores de fortificação e recuperações obtidas para a cafeína em amostras de pó de guaraná coletadas nos municípios de Campinas e Ribeirão Preto, entre dezembro de 2003 e janeiro de 2004.

\begin{tabular}{lcc}
\hline \multirow{2}{*}{ Cafeína/nível de fortificação $(\mathrm{mg} / 100 \mathrm{~mL})$} & \multicolumn{2}{c}{ Recuperação } \\
\cline { 2 - 3 } & média $(\%)^{\mathbf{1}}$ & $\mathrm{DP}$ \\
\hline 10 & 80,5 & 5,2 \\
15 & 71,5 & 2,1 \\
20 & 73,1 & 7,2 \\
\hline
\end{tabular}

${ }^{1}$ média de duas determinações; DP: Desvio-padrão

Tabela 2. Teores de cafeína $(\mathrm{mg} / \mathrm{g})$ em diferentes marcas de guaraná em pó coletadas nos municípios de Campinas e Ribeirão Preto, entre dezembro de 2003 e janeiro de 2004.

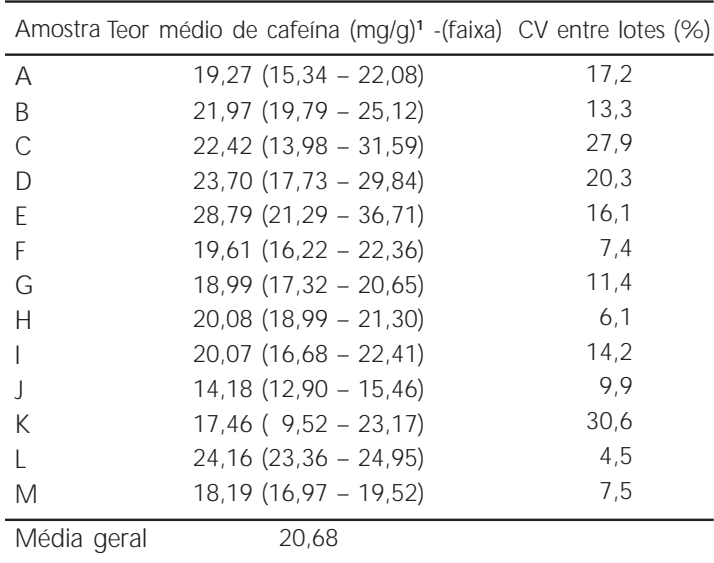

${ }^{1}$ M édia de três lotes; CV: Coeficiente de variação.
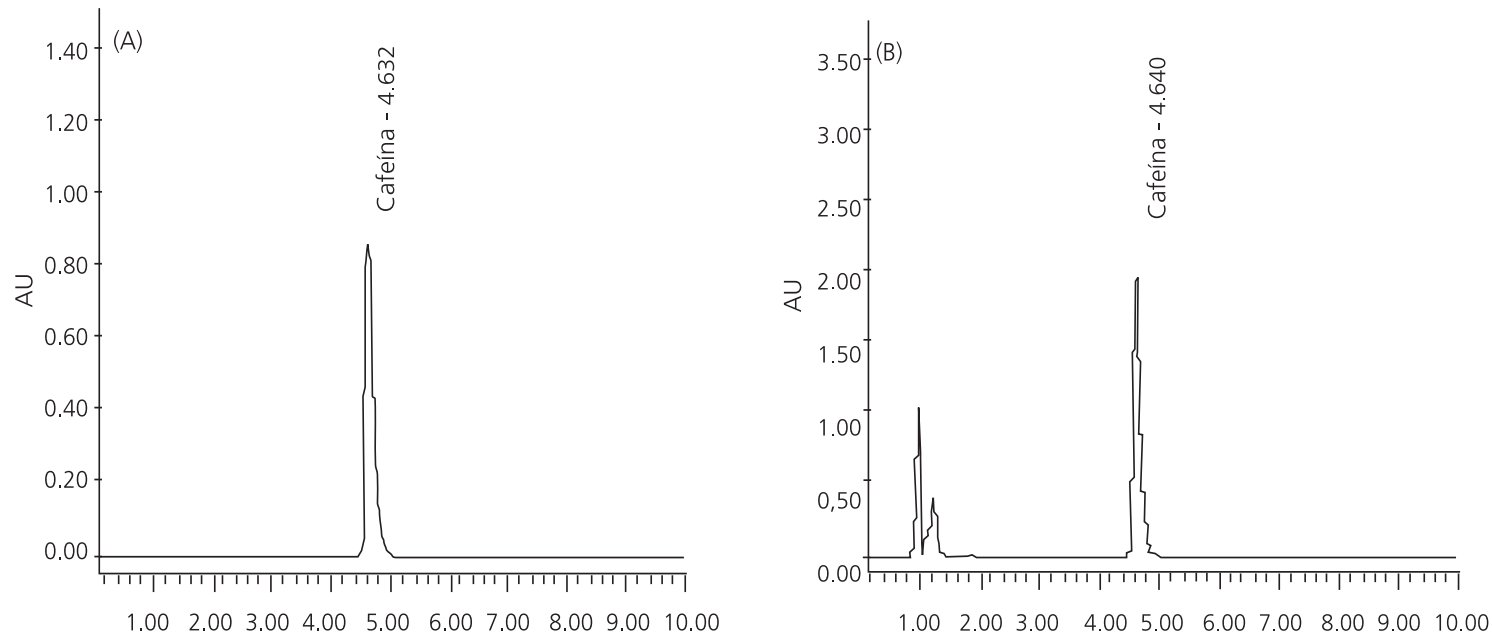

Figura 1. Cromatogramas por CLAE* referente (A) padrão de cafeína; (B) amostra de guaraná em pó. Coluna Novapack C18 (150 x 3,9mm d.i., partículas de $4 \mu \mathrm{m})$. Fase móvel: acetonitrila-água $(10: 90, v / \mathrm{v})$; vazão: $1,0 \mathrm{~mL} / \mathrm{min}$; volume de injeção: $20 \mu \mathrm{L} ;$ detector de arranjo de diodos (200-400nm) - cromatograma registrado a $272 \mathrm{~nm}$. Amostras coletadas nos municípios de Campinas e Ribeirão Preto, entre dezembro de 2003 e janeiro de 2004.

* cromatografia líquida de alta eficiência 
límpida, mostrando que o mesmo é um excelente clarificante para a remoção de proteínas solúveis e outras substâncias interferentes.

Como pode ser observado na Tabela 2, as quantidades de cafeína nas amostras de guaraná em pó variaram tanto entre marcas quanto entre lotes de uma mesma marca, sendo que os coeficientes de variação entre lotes situaram-se na faixa de $4,5 \%$ a $30,6 \%$. As diferenças apresentadas, possivelmente, se devem à procedência e processo a que a matéria-prima foi submetida. Empresas varejistas muitas vezes adquirem o pó de diferentes fornecedores e o embalam sob uma determinada marca comercial. Dessa forma, o produto final pode ser resultante de uma mistura de sementes oriundas de diferentes regiões produtoras do País.

Na literatura científica não existem dados disponíveis quanto à concentração de cafeína no guaraná em pó. Os dados encontrados são referentes apenas à sua presença em refrigerantes de guaraná $(1,1 \mathrm{mg} / 100 \mathrm{~mL})$ e xaropes $(<0,01 \mathrm{a}$ $86,40 \mathrm{mg} / 100 \mathrm{~mL})^{25,26}$. Dessa forma, em função do crescente consumo de guaraná em pó pela população brasileira nos últimos anos e sua associação com o consumo de demais produtos que contêm cafeína, a determinação da quantidade média deste alcalóide no produto em questão é importante para avaliar a contribuição do guaraná em pó como fonte de cafeína na dieta.

Ao comparar os níveis médios de cafeína encontrados no presente estudo com aqueles determinados por Camargo \& Toledo ${ }^{20}$ para o pó de café $(7,59 \mathrm{mg} / \mathrm{g})$, fonte tradicional de cafeína na dieta, verifica-se que cerca de $50 \%$ das amostras analisadas apresentaram teores de cafeína, aproximadamente, três vezes maior.

Dessa forma, um indivíduo, ao seguir a orientação do fabricante para o consumo diário de guaraná em pó, que é de 3 a $15 \mathrm{~g}$, poderá ingerir até $551 \mathrm{mg}$ de cafeína. Isso significa que um consumidor freqüente de guaraná em pó, independentemente de ingerir conjuntamente outras fontes de cafeína na dieta, poderá apresentar sintomas característicos do "cafeinismo", que, conforme o dito anteriormente, pode provocar ansiedade, inquietação, irritabilidade, tremores, perda de apetite tensão muscular e palpitações no coração.

Os resultados obtidos no presente estudo mostraram a grande variabilidade existente em relação ao teor de cafeína nas diferentes marcas de guaraná em pó disponíveis no comércio. Verificou-se também que, em média, a quantidade deste alcalóide no guaraná é maior do que no café. Entre os consumidores de guaraná em pó esse produto pode ser considerado como uma importante fonte da cafeína na dieta. Com isso, conclui-se que o consumo de guaraná em pó deve ser controlado, uma vez que controvérsias persistem quanto à dose segura de ingestão de cafeína.

\section{A GRA DECIMENTOS}

À Coordenadoria de Aperfeiçoamento de Pessoal de Nível Superior (Capes) e ao Conselho Nacional de Desenvolvimento Científico e Tecnológico (CNPq), pela concessão de auxílio financeiro à pesquisa.

\section{REFERÊ N C IA S}

1. Institute of Food Technologists. Caffeine. Food Technol. 1983; 37(4):87-91.

2. Couper-Smartt J, Couper-Smartt I. Caffeine consumption: a review of its use, intake, clinical effects and hazards. Food Technol Aus. 1984; 36(3): 131-4.

3. Leonard TK, Watson RR, Mohs ME. The effects of caffeine on various body systems: a review. J Am Diet Assoc. 1987; 87(8):1048-52.

4. Stavric B. Methylxanthines: toxicity to humans II. Caffeine. Food Chem Toxicol. 1988; 26(7):622-45.

5. Institute of Food Technologists. Evaluation of caffeine safety. Food Technol. 1988; 40(3):106-15.

6. Finnegan D. The health effects of stimulant drinks. Nutr Bull. 2003; 28(2):147-55.

7. Victor BS, Lubetsky M, Greden JF. Somatic manifestations of caffeinism. J Clin Psychiat. 1981; 42(5):185-8.

8. von Borstel RW. M Metabolism. Food Technol. 1983; 37(9):40-4. 
9. James JE. Caffeine and health. London: Academic Pres; 1991.

10. McKim EM, McKim WA. Caffeine: how much is too much? Can Nurse. 1993; 89(11):19-22.

11. Barone JJ, Roberts H. Caffeine consumption. In: Caffeine Workshop, 7. Santorini. Washington (DC): International Life Sciences Institute; 1993. cap.1:1-5.

12. Brasil. Ministério da Agricultura. Decreto $n^{\circ}$ 87.105. Normas de identidade, qualidade, embalagem, armazenamento e transporte do guaraná em grão, em bastão e em pó. Diário Oficial da União. 198219 abr.

13. Pinto MAR. Guaraná: alguns aspectos da produção e da comercialização. Rev Política Agrícola. 1998; 7(1):1-7.

14. Superintendência da Zona Franca de Manaus. Potencialidades Regionais: estudo da viabilidade econômica, Guaraná. M anaus: SUFRAMA; 2003.

15. Ashihara $\mathrm{H}, \mathrm{Crozier} A$. Caffeine: a well known but little mentioned compound in plant science. Trends Plant Sci. 2001; 6(9):407-13.

16. Madison BL, Kozarek WJ, Damo CP. High-pressure liquid chromatography of caffeine in coffee. J Assoc Off Anal Chem. 1976; 59(6):1258-61.

17. Ashoor SH, Seperich GJ, Woodrow CM, Welty J. High performance liquid chromatographic determinations of caffeine in decaffeinated coffee, tea, and beverages products. J Assoc Off Anal Chem. 1983; 66(3):606-9.

18. Blauch JL, Tarka SM. HPLC determination of caffeine and theobromine in coffee, tea, and instant hot cocoa mixes. J Food Sci. 1983; 48(3):745-50.

19. Galasko GTF, Furman KI, Alberts E. The caffeine contents of non-alcoholic beverages. Food Chem Toxicol. 1989; 27(1):49-51.
20. Camargo MCR, Toledo MCF. Teor de cafeína em cafés brasileiros. Ciênc Tecnol Aliment. 1998; 18(4):421-4.

21. Naik JP, Nagalakshmi S. Determination of caffeine in tea products by an improved high performance liquid chromatography method. J Agric Food Chem. 1997; 45(10):3973-5.

22. Carlson M, Thompson RD. Liquid chromatographic determination of methylxanthines and catechins in herbal preparations containing guaraná. J AOAC Inter. 1998, 81(4):691-701.

23. Casal S, Oliveira MBPP, Alves MR, Ferreira MA. Discriminate analysis of roasted coffee varieties for trigonelline, nicotinic acid and caffeine content. J Agric Food Chem. 2000; 48(8):3420-4

24. Naik JP. Improved high performance liquid chromatography method to determine theobromine and caffeine in cocoa and cocoa products. J Agric Food Chem. 2001, 49(8): 3579-83.

25. Aquino FWB, Amorim AGN, Prata LF, Nascimento RF. Determinação de aditivos, aldeídos furânicos, açúcares e cafeína em bebidas por cromatografia líquida de alta eficiência: validação de metodologias. Ciênc Tecnol Aliment. 2004; 24(1):32-8.

26. Camargo MCR, Toledo MCF. HPLC determination of caffeine in tea, chocolate products and carbonated beverages. J Food Sci Agric. 1999; 79(13):1861-4.

27. Miller JC, Miller JN. Statistics for Analytical Chemistry, Chichester: Ellis Horwood Limited; 1993.

Recebido em: 30/9/2005

Versão final reapresentada em: 7/8/2006 Aprovado em: 19/10/2006 